\title{
Chemistry in Four Dimensions
}

Jan C. A. Boeyens

\begin{abstract}
Some chemical phenomena, awkward to rationalize, are argued to originate in the four-dimensional nature of matter in curved space-time. The problem is traced back to the separation of space and time variables in the analysis of fourdimensional events. Although mathematically sound, this operation is not physically valid. It destroys the essential non-classical entanglement of space and time, which is recognized in relativistic theory, but not in quantum mechanics. We show that without this approximation the state functions of quantum theory have the same quaternion structure that describes Lorentz transformation and spin. Hypercomplex formulation of four-dimensional motion eliminates several bothersome concepts, such as wave-particle duality and probability density, by providing the logical basis for non-zero commutators in non-classical systems. It shows why chiral states are undefined in quantum theory and why many solid-state transitions appear to be sterically forbidden. A brief introduction to hypercomplex algebra is given as an Appendix.

Keywords: d'Alembertian, Dirac's equation, harmonic function, spin function, quaternion
\end{abstract}

\section{Introduction}

Several generations of chemists have been conditioned to accept the notorious discrepancy between the theory and practice of chemistry as the unquestionable norm. Sterically forbidden molecular rearrangements and phase transformations are routinely reported without comment and the flow of electronic particles, postulated to rationalize the course of chemical reactions, is never subjected to critical scrutiny. In reality, practising chemists design their experiments in terms of the 19th century

Jan C. A. Boeyens

Unit for Advanced Scholarship, University of Pretoria, South Africa,

e-mail: jan.boeyens@up.ac.za 
notions of chemical affinity, never adequately explained by 20th century theories. The innocent belief that quantum physics explains "all of chemistry" is, like the rest of quantum theory, obediently respected as just another of its deep inscrutable mysteries.

The reluctance to abandon dogmatic theory often results in the introduction of secondary ad hoc explanations to cover up any cracks in the theory, as they occur. A prime example occurs in the quantum theory of elemental periodicity. Based on the wave-mechanical ordering of electronic energy levels in an isolated $\mathrm{H}$ atom, a logical Aufbau procedure for many-electron atoms suggests itself. Elemental ordering in shells, consisting of $2 n^{2}$ successive atoms, for integral $n>0$, is predicted. This pattern breaks down at $n=3$. In order to rescue the theory a poorly explained effect, ascribed to interelectron repulsion is conjectured, without modification of the basic theory. Even if the only effect of the additional assumption were the splitting of energy sub-levels whereby, for instance, the $4 s$ sub-level occurs at a lower energy than $3 d$, it still fails to account for the observed periodicity. Instead of the expected $4 s^{1 \rightarrow 2} 3 d^{1 \rightarrow 10}$, the sequence $4 s^{1 \rightarrow 2} 3 d^{1 \rightarrow 8}\left(3 d^{10} 4 s^{1 \rightarrow 2}\right)$ is observed. Contrary to Aufbau philosophy the interpolated transition series is almost uniformly characterized by a $4 s^{2}$, rather than $3 d$, valence shell. Arbitrary new concepts, such as the degree of orbital penetration towards the nucleus, without any wave-mechanical basis, are invoked next, without improving the theory in any way. A more appropriate response would have been to admit failure of the $\mathrm{H}$ model, applied to non-hydrogen atoms.

The unjustified confidence in wave mechanics to account for elemental periodicity encouraged the belief in quantum theory to explain all chemistry, albeit with the aid of supercomputers - another unfulfilled dream. The cruellest reality for chemists has perhaps been the consistent failure to identify the quantum-mechanical basis of the three-dimensional structure of molecules, substantiated by thousands of independent observations, and despite massive computational efforts. This negative result provides a significant clue to account for the failure of quantum theory in the description of chemical systems.

One of the more successful devices to reconcile chemical behaviour with quantum theory was the proposed definition of atomic orbitals to regulate the distribution of electrons on both atoms and molecules. A minor irritant in this application is the complex nature of the relevant wave functions that underlie the definition of atomic orbitals. As these complex functions invariably occur in orthogonal pairs, real functions can be constructed by suitable linear combinations of these pairs. The same strategy is used in all quantum-chemical computations in order to avoid complex variables. This strategy comes at an exorbitant price.

A wave-mechanical model of the $\mathrm{H}$ atom describes an electron in terms of three quantum numbers. However, in order to account for atomic spectra it is necessary to assume that the extranuclear electrons are not all concentrated at the lowest energy level, but distributed over several levels as stipulated by a fourth quantum number, postulated to represent a two-level spin system that obeys an exclusion principle. The strict consequence of this observation is that the orbitals of a three-fold degenerate level must have the third quantum number with values of $m_{l}=-1,0,1$, which eliminates the possibility of three real functions (All $m_{l}=0$ ). The simple conclusion 
is that any computational scheme that operates exclusively on real variables cannot be considered to be quantum mechanical, but rather as strictly classical.

The pioneers of quantum theory were unprepared for the discovery of two related, but unforeseen, new effects - the appearance of complex variables as an essential feature of the theory and pairs of conjugate variables that do not commute; a stark departure from classical mechanics. The appearance of complex variables is now readily appreciated, even in two-dimensional harmonic functions, defined by the equation:

$$
\frac{\partial^{2} \Phi}{\partial x^{2}}+\frac{\partial^{2} \Phi}{\partial y^{2}}=0
$$

One method to solve this equation is by assuming the potential function to be the product of two variable functions of $x$ and $y$ respectively; i.e.:

$$
\Phi=X \cdot Y
$$

which on differentiation gives

$$
\frac{1}{X} \frac{\partial^{2} X}{\partial x^{2}}+\frac{1}{Y} \frac{\partial^{2} Y}{\partial y^{2}}=0
$$

The two terms are functions of independent variables and, in order to satisfy (1) must be independent of both variables and therefore equal to a constant, i.e.

$$
\frac{1}{X} \frac{\partial^{2} X}{\partial x^{2}}=k_{x}^{2} \quad ; \quad \frac{1}{Y} \frac{\partial^{2} Y}{\partial y^{2}}=k_{y}^{2} .
$$

To satisfy the implied condition, $k_{x}^{2}+k_{y}^{2}=0$, either $k_{x}=k_{y}=0$, or $i k_{x}=k_{y}$, such that

$$
\Phi=c e^{ \pm k(x+i y)}
$$

which describes a rotation in complex notation, exactly as required by wave mechanics.

In three-dimensional formulation the condition $k_{x}^{2}+k_{y}^{2}+k_{z}^{2}=0 \quad$ or $\quad(A+i B)^{2}$ is satisfied, without loss of generality on setting $k_{z}=0, i k_{x}=k_{y}$, to describe a threefold degenerate state by the magnetic quantum number $m_{l}=0, \pm 1$. Equating all constants to zero, by the mathematical separation of the physically entangled $x$ and $y$ coordinates, not only avoids the use of complex functions, but also destroys the ability to describe the angular momentum of the system. The one-dimensional projection appears as harmonic oscillation, e.g.

$$
\frac{\partial^{2} X}{\partial x^{2}}=k^{2} X \quad, \quad X=a e^{ \pm k x}
$$

which is an incomplete description of the complex rotation.

We have reached the uncomfortable conclusion that our trusted three-dimensional wave equation, interpreted as a projection from four-dimensional space-time must 
have the same defects as an harmonic oscillator when being considered a model of complex rotation.

\section{Four-dimensional Motion}

Motion in four dimensions proceeds against the gradient of a scalar function $\Phi$,

$$
\square \Phi=\sum_{\mu=0}^{3} \frac{\partial \Phi}{\partial x_{\mu}}=\sum \partial \Phi_{\mu} \equiv\left(\frac{1}{i c} \frac{\partial}{\partial t}+\frac{\partial}{\partial x}+\frac{\partial}{\partial y}+\frac{\partial}{\partial z}\right) \Phi
$$

and the potential field is described by the d'Alembertian:

$$
\begin{aligned}
\square^{2} \Phi & =\left(\frac{\partial^{2}}{\partial x_{0}^{2}}+\frac{\partial^{2}}{\partial x_{1}^{2}}+\frac{\partial^{2}}{\partial x_{2}^{2}}+\frac{\partial^{2}}{\partial x_{3}^{2}}\right) \Phi=0 \\
& \equiv\left(\nabla^{2}-\frac{1}{c^{2}} \frac{\partial^{2}}{\partial t^{2}}\right) \Phi=0
\end{aligned}
$$

Equation (2) is recognized as Laplace's equation in four dimensions and in alternative form is known as the wave equation in 3-dimensional space.

The mathematical procedure of solving (2) is by treating $\Phi$ as the product function

$$
\Phi=X_{0}\left(x_{0}\right) \cdot X_{1}\left(x_{1}\right) \cdot X_{2}\left(x_{2}\right) \cdot X_{3}\left(x_{3}\right) .
$$

By substituting the four factors,

$$
\frac{\partial^{2} \Phi}{\partial x_{0}^{2}}=\frac{\partial^{2} X_{0}}{\partial x_{0}^{2}}\left(X_{1} X_{2} X_{3}\right), \quad \text { etc. }
$$

into (2) and dividing by $\Phi$,

$$
\frac{1}{\Phi} \square^{2} \Phi=\frac{1}{X_{0}} \frac{\partial^{2} \Phi}{\partial x_{0}^{2}}+\frac{1}{X_{1}} \frac{\partial^{2} \Phi}{\partial x_{1}^{2}}+\frac{1}{X_{2}} \frac{\partial^{2} \Phi}{\partial x_{2}^{2}}+\frac{1}{X_{3}} \frac{\partial^{2} \Phi}{\partial x_{3}^{2}}=0 .
$$

Each term is a function of a single variable and (3) can only remain valid if each term is independent of all variables and equal to a constant. Each term can hence be written in the form of a one-dimensional Helmholtz equation:

$$
\frac{d^{2} X}{d x^{2}}-k^{2} X=0
$$

in which $X=a e^{ \pm k x}$. For $X=T(t)$ the equation

$$
\frac{1}{c^{2}} \frac{d^{2} T}{d t^{2}}+k^{2} T=0
$$


writing $\omega=c k$, yields $T(t)=a \exp ( \pm i \omega t)$, in which $\omega$ is interpreted physically as an angular frequency.

Each term in (3) requires an arbitrary constant to generate the overall solution $\Phi=A \exp \left(k_{0} x_{0}+k_{1} x_{1}+k_{2} x_{2}+k_{3} x_{3}\right)$. These four constants are not independent and must satisfy the general condition: $\sum k_{\mu}^{2}=0$, imposed by (3) and (4). The most general solution of $\square^{2} \Phi=0$ is therefore a function that depends equally on all four coordinates, rather than the product of four linear functions. The four-dimensional Laplacian, $\square^{2} \Phi=0$ describes the state of potential balance along the curved Riemannian manifold. Minkowski space-time, tangent to the manifold, describes a local pseudo-Euclidean approximation. By separating space and time variables the Laplacian reduces to a wave equation (2a) in Euclidean space. This equation is the basis of wave mechanics, which further separates space and time variables into space-like and time-like equations.

It is at this point that wave mechanics moves out of Minkowski space. Away from the assertion that space-time is characterized by the harmonics of a four-dimensional Laplacian, visualized as long-wavelength undulation; like a wave field in Minkowski space. In wave-mechanical approximation the time and space variables (collectively represented as $x$ ) are separated by definition of the product function

$$
u(x, t)=f(x) \cdot e^{-i \omega_{0} t}
$$

to yield

$$
\nabla^{2} \psi+\left(\frac{\omega_{0}}{c}\right)^{2} \psi=0
$$

and

$$
\left(\nabla^{2}+\frac{i \omega_{0}}{c^{2}} \frac{\partial}{\partial t}\right) \Psi=0 .
$$

By de Broglie's postulate, the wave vector becomes

$$
k=\frac{\omega_{0}}{c}=\frac{2 \pi}{\lambda}=\frac{p}{\hbar}
$$

whereby (5) and (5a) reduce to the familiar Schrödinger equations, which provide a good practical approximation within the locally perceived three-dimensional space, but the holistic entanglement of space-time coordinates is lost.

Developed into a theory of atomic stability the equilibrium condition, $d E / d r=0$, defines the so-called stationary states of the hydrogen atom as a function of the total electronic energy,

$$
E=\frac{p^{2}}{2 m}-\frac{e^{2}}{r} \quad \text { (in esu). }
$$

Considerd as a standing de Broglie wave of wavelength $n \lambda=2 \pi r_{n}$, the distance between proton and electron follows as 


$$
r_{n}=\frac{(n \hbar)^{2}}{m e^{2}}, \quad \text { i.e. } \quad E_{n}=-\frac{e^{2}}{2 r}=-\frac{m e^{4}}{2(n h)^{2}}=-\frac{R h c}{n^{2}}
$$

This inverse square relationship, which assumes a spherically symmetrical standing electron wave, is the fundamental equation of atomic spectroscopy and nonrelativistic wave mechanics. In four-dimensional space-time, especially in a nonzero gravitational field, the assumption is not strictly valid and the proportionality factor, $R_{n}$ may vary with $n$.

The mass variable is a strictly empirical assumption that only acquires meaning in non-Euclidean space-time on distortion of the Euclidean wave field defined by equation (2). The space-like equation (5), known as Schrödinger's time-independent equation is not Lorentz invariant. It is satisfied by a non-local wave function which, in curved space, generates time-like matter-wave packets, characterized in terms of quantized energy and three-dimensional orbital angular momentum. The fourdimensional aspect of rotation, known as spin, is lost in the process and added on by assumption. For macroscopic systems the wave-mechanical quantum condition $\hbar \omega=E-V$ is replaced by Newtonian particle mechanics, in which $E=\frac{1}{2} m v^{2}+V$. This condition, in turn, breaks down as $v \rightarrow c$.

Wave mechanics, and particle mechanics, formulated to describe motion in threedimensional space are both incomplete by their failure to account for spin and relativistic effects. The common defect in both formulations lies in the unphysical separation of space and time variables. The proper procedure requires hypercomplex solutions of (2), which describe motion in four-dimensional space-time ${ }^{1}$.

Although hypercomplex solution of (2) has never been achieved, a conditional covariant form of the wave equation was proposed by Dirac [1] on assuming spin matrices as possible solutions. The procedure consisted of modifying Schrödinger's equation

$$
i \hbar \frac{\partial \Psi}{\partial t}=H \Psi
$$

which is linear in $\partial / \partial t$, by inserting the relativistic Hamiltonian

$$
H=\left(p^{2} c^{2}+m_{0}^{2} c^{4}\right)^{\frac{1}{2}} .
$$

To ensure Lorentz invariance the Hamiltonian should also be linear in space derivatives, $-\hbar i p_{k}=\partial / \partial x_{k} \quad(k=1,2,3)$, such that

$$
\begin{gathered}
H=\sum_{k=1}^{3} \alpha_{k} c p_{k}+\beta m_{0} c^{2}, \quad \text { i.e. } \\
i \hbar\left[\frac{\beta}{c} \frac{\partial}{\partial t}+\sum_{k=1}^{3} \beta \alpha_{k} \frac{\partial}{\partial x_{k}}\right] \Psi=m_{0} c \Psi .
\end{gathered}
$$

\footnotetext{
${ }^{1}$ For the benefit of those readers who are not familiar with hypercomplex numbers and quaternions an elementary introduction is provided as an Appendix to this paper.
} 
With $\gamma^{0}=\beta, \gamma^{k}=\beta \alpha_{k}, x_{0}=c t$ the equation

$$
i \hbar\left(\gamma^{0} \frac{\partial}{\partial x_{0}}+\gamma^{1} \frac{\partial}{\partial x_{1}}+\gamma^{2} \frac{\partial}{\partial x_{2}}+\gamma^{3} \frac{\partial}{\partial x_{3}}\right) \Psi=m_{0} c \Psi
$$

is Lorentz invariant and commonly transformed, using units of $\hbar=c=1$ and the Einstein summation convention, to read

$$
\left(i \gamma^{\mu} \partial_{\mu}-m\right)=0
$$

These conditions are fulfilled by assuming coefficients of the form

$$
\alpha_{k}=\left[\begin{array}{ll}
0 & \sigma_{k} \\
\sigma_{k} & 0
\end{array}\right] \quad, \quad \beta=\left[\begin{array}{rr}
I & 0 \\
0 & -I
\end{array}\right]
$$

where $\sigma_{k}$ are Pauli matrices and $I$ is the $2 \times 2$ unit matrix. As before, the spin variables are therefore introduced empirically.

The most significant difference of Dirac's results from those of the non-relativistic Pauli equation is that the orbital angular momentum and spin of an electron in a central field are no longer separate constants of the motion. Only the components of $\mathbf{J}=\mathbf{L}+\mathbf{S}$ and $\mathbf{J}^{2}$, which commute with the Hamiltonian, emerge as conserved quantities [1]. Dirac's equation, extended to general relativity by the method of projective relativity [2] automatically ensures invariance with respect to gauge, coordinate and spinor transformations, but has never been solved in this form.

The surprising implication is that Dirac's equation does not allow of a selfconsistent single-particle interpretation, although it has been used to calculate approximate relativistic corrections to the Schrödinger energy spectrum of hydrogen. The obvious reason is that a 4D point particle is without duration and hence undefined. An alternative description of elementary units of matter becomes unavoidable. Prompted by such observation Dirac [3] re-examined the classical point model of the electron only to find that it has three-dimensional size, with an interior that allows superluminal signals. It all points at a wave structure with phase velocity $v_{\phi}>c$.

The equation $\square^{2} \Phi=0$ has unitary quaternion solutions of the form

$$
\Phi=e^{\theta(i \alpha+j \beta+k \gamma)}=\cos \theta+\sin \theta(i \alpha+j \beta+k \gamma)
$$

which represent the $S U(2)$ rotational Lie group. The four-dimensional eigenfunction is the proper spin function. With $\theta=\omega t$ it is the state function that describes total angular momentum $\mathbf{J}=\mathbf{L}+\mathbf{S}$. For $i \alpha+j \beta+k \gamma=i$, it describes spin only. Finally, with $\beta=\gamma=0$, it defines spin in polar projection $\left(s= \pm \frac{1}{2}\right)$, as shown in more detail below. Alternatively $\Phi$ is the state function of an electromagnetic photon.

With $\cos \theta=0 \Phi$ could represent the space-like state function of electric charge, known as the angular-momentum function, described by the quantum number $l$ in Schrödinger notation, also known as the three-dimensional spherical harmonics.

The most general rotation of a four-vector represented by $q=w+i x+j y+k z$ is described by the equation 


$$
q^{\prime}=e^{\theta(i \alpha+j \beta+k \gamma)} q e^{-\varphi(i \lambda+j \mu+k v)}
$$

where $\alpha^{2}+\beta^{2}+\gamma^{2}=\lambda^{2}+\mu^{2}+v^{2}=1$. When both $\theta$ and $\varphi$ are imaginary angles, the transformation corresponds to the Lorentz transformation $[4,5]$. This is mathematical proof that quantum theory and special relativity originate from the same basis, as the most general solution of (2) must obviously be a quaternion function.

\section{Spherical Rotation and Spin}

The quaternion that describes rotation through $\theta$ about the $x$-axis, follows as

$$
e^{i(\theta / 2)}=\cos (\theta / 2)+i \sin (\theta / 2)
$$

This hypercomplex number is given in matrix form by

$$
q=\left(\begin{array}{cc}
0 & e^{-i \theta / 2} \\
e^{i \theta / 2} & 0
\end{array}\right)
$$

and rotation of $2 \omega t$ in time, by

$$
\left(\begin{array}{cc}
0 & e^{-i \omega t} \\
e^{i \omega t} & 0
\end{array}\right)
$$

This matrix operates on a two-level object, called a spinor, e.g. $\left\{\phi_{1}, \phi_{2}\right\}$. A spinor system that moves in some general direction, $x$, say:

$$
\Phi=\left(\begin{array}{cc}
0 & e^{-i(\omega t-k x)} \\
e^{i(\omega t-k x)} & 0
\end{array}\right)\left(\begin{array}{l}
\phi_{2} \\
\phi_{1}
\end{array}\right) \equiv\left(\begin{array}{l}
\phi_{1} e^{-} \\
\phi_{2} e^{+}
\end{array}\right)
$$

(in shorthand notation) is shown to satisfy Schrödinger's [6] and Dirac's [7] equations, by forming the derivatives:

$$
\frac{\partial \Phi}{\partial t}=i \omega\left(\begin{array}{c}
-\phi_{1} e^{-} \\
\phi_{2} e^{-}
\end{array}\right), \quad \frac{\partial \Phi}{\partial x}=i k\left(\begin{array}{c}
\phi_{1} e^{-} \\
\phi_{2} e^{+}
\end{array}\right), \quad \frac{\partial^{2} \Phi}{\partial x^{2}}=k^{2}\left(\begin{array}{c}
-\phi_{1} e^{-} \\
\phi_{2} e^{+}
\end{array}\right)
$$

from which follows that $\quad \frac{1}{i \omega} \frac{\partial \Phi}{\partial t}=\frac{1}{k^{2}} \frac{\partial^{2} \Phi}{\partial x^{2}}$.

Equating $m=\hbar k^{2} / 2 \omega$, the equation

$$
-i \frac{\partial \Phi}{\partial t}=\frac{\omega}{k^{2}} \nabla^{2} \Phi=\frac{\hbar}{2 m} \nabla^{2} \Phi
$$

in three dimensions, is identical to Schrödinger's equation or its complex conjugate, and describes both matter and antimatter, each with the spin states $\left\{\phi_{1}, \phi_{2}\right\}$. Writing 
the wave vector $k=2 \pi / \lambda=p / \hbar$, with de Broglie, the quantum condition $\hbar \omega=$ $p^{2} / 2 m$ follows directly.

The common assertion that electron spin is a strictly relativistic effect [8] needs modification. As shown before [9] a linearized Schrödinger equation, first order in all space-time derivatives implies the Pauli equation [10] with the correct value of the electron gyromagnetic ratio. The 1/2-spin appears, not as a relativistic effect, but because of the implied four-dimensional space-time. It has the significant advantage that the spin degrees of freedom are contained in the theory from the beginning, without being added $a d$ hoc. However, the spin and angular-momentum variables remain separated.

Since there is no geometrical understanding of spin in three-dimensional space it is not reflected in standard wave mechanics. A four-dimensional quaternion function, on the other hand, contains a four-component spinor as temporal characteristic, entangled with the space variables. Spin is generated in four-dimensional quaternion rotation, which is intrinsically different from the axial rotation of three-dimensional space, and is also described as spherical rotation ${ }^{2}$ [7]. Because rotation in spin space is governed by one-half the angles of rotation in ordinary space, it has the special property that any entanglement of the spinor with the supporting medium, which develops during rotation of $2 \pi$, spontaneously disentangles after a further $2 \pi$ rotation. This is the result of the half-angle operation of quaternion rotation. It causes a periodic fluctuation in the medium (vacuum) that surrounds the spinor, and is observed as spin. The undulation with $\lambda=2 \pi$ on the surface of the unit sphere describes spin as $m \omega=\hbar / 2$, half a unit of angular momentum.

Each spin $\left(\begin{array}{c}e^{i \omega t} \\ 0\end{array}\right)$ has an inverse state $\left(\begin{array}{c}0 \\ i \omega t\end{array}\right)$ and an antispin $\left(\begin{array}{c}e^{-i \omega t} \\ 0\end{array}\right)$. The products

$$
\left(\begin{array}{ll}
0 i \omega t) \\
e^{i \omega t} \\
0
\end{array}\right)=0 \quad, \quad\left(e^{-i \omega t} 0\right)\left(\begin{array}{c}
e^{i \omega t} \\
0
\end{array}\right)=1
$$

show that spin pairing yields a boson of spin zero, while the annihilation of spin and antispin produces a photon of spin $\hbar$ and which satisfies the wave equation (2a). The condition $c=\sqrt{E / 2 m},(V=0)$, now implies conversion of the total mass of matter and antimatter into a photon of energy $E=2 m c^{2}=\hbar \omega$.

It is ironic that spin, which is the only non-classical attribute of quantum mechanics, is absent from the pioneering formulations of Heisenberg and Schrödinger. Even in Dirac's equation the appearance of spin is ascribed by fiat to Lorentz invariance, without further elucidation. In reality, both Lorentz invariance and spin, representing relativity and quantum mechanics, respectively, are properties of the quaternion field that underpins both theories.

\footnotetext{
2 The relationship between spherical rotation and quaternions is visualized pictorially by Kauffman[11]
} 


\section{The Physical Model}

Within the time-like domain of Minkowski space the gravitational field dominates and a quantum-potential field dominates in the space-like domain. Electromagnetic bosons, which occur in the interface, exhibit both time-like and space-like behaviour. Events close to, or in, the interface show both quantum and relativistic behaviour. There is only one classical-non-classical limit as quantum mechanics and relativity appear as a single theory. Confusion sets in with failure to distinguish between waves and particles as elementary entities and with the possibility of massive objects moving faster than light in the vacuum. It is the unphysical identification of elementary point particles with quantum waves that gives rise to the spurious concepts of wavicle, quantum uncertainty, probability density and infinite self-energy. Elementary wave structures exist without such complications. It is instructive to note that the authoratitive exposition of Unsöld [12] does not involve probability density.

Standing waves, like gas molecules, exert pressure on the walls of a container. With the container at rest or in uniform motion the force exerted on any wall is balanced by that exerted on the opposite wall. If the container is accelerated radiation reflected off the rear wall gains more momentum than that lost by the radiation which reflects off the front wall. It has been demonstrated $[13,14,16]$ that radiation exerts a net force, which opposes an applied force on the container, such that

$$
\left(m+\frac{E_{R}}{c^{2}}\right) a=F .
$$

The radiant energy adds an effective inertial mass $E_{R} / c^{2}$ to the mass of the container. In the same way the total mass of an electron may be interpreted as deriving from internal motion in a phase-locked cavity.

Plane waves with phase velocity $v_{\phi}$ form wave packets with group velocity $v_{g}$, such that $v_{\phi} v_{g}=c^{2}\left(=1 / \varepsilon_{0} \mu_{0}\right)$. The phase and group velocities are the same only for electromagnetic waves with $v_{\phi}=c$. To describe the inertial properties of a wave packet a mass variable is introduced [17]. A group velocity $v_{g} \lessgtr c$ defines matter waves as described by the Elbaz [18] equation:

$$
\nabla^{2} u \pm\left(\frac{m_{0} c}{\hbar}\right)^{2} u=\frac{1}{c^{2}} \frac{\partial^{2} u}{\partial t^{2}}
$$

This is interpreted to show that the curving of space produces time-like $\left(v_{g}<c\right)$ as well as space-like $\left(v_{g}>c\right)$ wave packets with real $(\mathrm{m})$ and imaginary $(\mathrm{im})$ mass [19], respectively known as bradyons and tachyons.

Equation (8), second-order in time, has solutions $u(t)$ and $u(-t)$, defining matter and antimatter waves respectively. Of the four possible forms only bradyonic matter waves are directly observed in time-like tangent space. Other types of wave are encountered in sub-atomic quantum systems. 
The formation of wave packets depends on the interaction of a complementary bradyon-tachyon pair. The bradyonic group velocity corresponds to the de Broglie wavelength of the packet, $\lambda_{d B}=h / m v_{g}$. The tachyonic component defines the internal structure of the wave packet with Compton wavelength $\lambda_{C}=h / m c$. The two components are said [20] to be trapped in a relativistically invariant way. We note that $v_{b} v_{t}=c^{2}=1 / \varepsilon_{0} \mu_{0}$, where the group velocity of the tachyon $v_{t}$ matches the phase velocity of the bradyon. Such a wave-packet is not dispersive. Essentially the same model, developed from another perspective, is described by Milo Wolff [21]. An elementary unit of charge (e.g. electron) is considered here as a standing wave packet created by the interaction between a pair of time-symmetric spherical scalar waves: a retarded wave radiated by the electron, in balance with an advanced wave that represents the resultant as the radiation from all other sources in the cosmos interferes. This is Mach's principle in wave formalism.

Variability in the internal structure of elementary wave packets is responsible for the appearance of protons, neutrons and electrons [22], which interact by the exchange of intermediate massless photons. Chemical interactions, all of this type [6], are responsible for the growth of massive structures, which on the macro scale interact gravitationally.

All debates on the interpretation of quantum mechanics must end in confusion, unless the classical and non-classical models of the world are clearly distinguished. The classical model is based on the assumption that persistent fragmentation of matter terminates in a set of elementary particles that resist further subdivision, but retain the innate quality to predict the behaviour of matter in the bulk. A non-classical alternative starts at the other extreme with a featureless plenum that develops periodic wave structures in a topologically closed universe. In projective relativity [23] there is

... no such thing as a body in space, but matter is an aspect of the space-time structure.

These elementary waves coalesce into bigger units that exhibit all the known properties of ponderable matter.

Classical mechanics analyzes the interaction between particles, and non-classical mechanics should study the interaction between wave structures. We repeat that the two models do not refer to classical and non-classical domains - they both model the same world, but from different points of view. It so happens that at different levels of aggregation one or the other provides a more convenient description. Attempts to describe classical structures non-classically, or vice versa, inevitably end up with illogical conclusions.

Physics has the dilemma of irrefutable evidence for a four-dimensional world, but a genetic inability among physicists to visualize more than three dimensions. It is therefore not surprising to find that those instances in which reality is badly distorted in three-dimensional projection, inevitably lead to convoluted theories, bordering on the supernatural. Quantum mechanics is a prime example of such a theory. It was inspired by experimental results that defied explanation based on classical theory. It was first recognized in the study of microphysical systems, which in time came to 
be seen as deviating from the classical and therefore subject to a new theory, without relevance in macrophysics.

A more plausible interpretation is that the motion of ponderous objects, projected into tangent three-dimensional space, differs imperceptably from four-dimensional reality in the local environment where a classical description suffices. It only becomes an issue for fast-moving objects and where particle mass approaches zero. The real meaning of both relativity and quantum theory is obscured by their formulation as alternatives to Newtonian mechanics that kick in at some classical limit.

A notable difference between three- and four-dimensional formulation of quantum mechanics occurs in their commutation properties. In $\mathbb{R}^{3}$ commutators that involve a time-dependent variable are found to be non-zero. For instance

$$
[p, q]=(p q-q p)=i \hbar \quad, \quad\left[J_{x}, J_{y}\right]=i \hbar J_{z}, \text { etc } .
$$

Uncertainty relationships such as $\Delta p \Delta q>\hbar, \Delta E \Delta t>\hbar$, etc, derive directly from these commutation rules [24].

The unexpected appearance of complex operators is also associated with nonzero commutators and reflects the essential two-dimensional representation in $\mathbb{M}^{2}$ Minkowski space-time. In four-dimensional space-time, $\mathbb{H}^{4}$, all commutators are non-zero, as appropriate for wave motion of both quantum and relativity theories. An important consequence is that local observation has no validity on global extrapolation, as evidenced by the appearance of cosmical redshifts in the curved manifold and the illusion of an expanding universe.

\section{The Chemical Model}

Chemical theory, if anything, is distorted even more than physics on projection from four-dimensional space-time. In electromagnetic and other field theories gauge particles have mathematically assigned phase factors, which in chemistry is simulated as probability density. Whereas the purely mathematical symbolism suffices as working models in particle physics, chemistry has the more stringent demand to deal with extended three-dimensional entities. Even at its lowest level the known chemical function of an electron, defined as a structureless point particle, becomes incomprehensible.

The analysis of dynamic systems in terms of point particles originated with Newton, but the context in which the concept was introduced has been ignored and forgotten. Newton was concerned with the motion of heavenly bodies and their mutual interaction. To avoid the complication that the moon's effect at the proximal and distal surfaces of the earth cannot be the same, the total interaction was assumed mathematically equivalent to the attraction between objects with all mass concentrated at their respective centres of gravity, which is a point. Earth and moon are therefore modelled as interacting mass points. What works for planets also works for apples and electrons. In practical application reference to the centre of gravity 
was made less frequently and, in time, the actual nature of elementary units of matter as mass points became generally accepted as physical reality.

When the wave nature of electrons was discovered experimentally Newton's perception of mass points as centres of gravity had been inactive for so long that, instead of a wave packet with a centre of gravity, an electron was considered as the physical union of a particle and a wave. In this approach particle nature is considered to be of primary importance and wave nature is simulated by a probability function that specifies particle coordinates. This interpretation was pioneered by de Broglie's postulate [25] of a particle piloted by a wave. However, the more natural wave model does the opposite and follows the motion of a wave packet in terms of its centre-ofgravity coordinates. The photoelectric and Compton effects can then be understood as interaction between waves, rather than particles, as pointed out by Schrödinger [26]. As stated in the Abstract of [26]:

A definite $\psi$-distribution in configuration space is interpreted as a continuous distribution of electricity (and electric current density) in actual space.

Unforunately this level-headed interpretation was never generally accepted. As remarked by David Bohm [27]:

The current formulation of quantum mechanics must be regarded merely as a statistical algorithm, which provides no conceptual structure in terms of which the movement of individual systems can be understood.

It is only the theory of general relativity that provides any guidance towards an understanding of the elementary nature of matter, via Einstein's field equations, conveniently condensed into the form

$$
G_{\mu v}=k T_{\mu v} \quad, \quad \mu, v=0,3
$$

which balances the tensor of space-time curvature against the stress tensor of the matter-energy field. The vital assertion is that a vanishing curvature tensor, which implies flat Euclidean space, demands the disappearance of all matter. It is unequivocally inferred that matter appears as a result of the curving of space-time. By the minimal assumption, known as Occam's razor, matter must be identified as a distortion of curved space-time.

As a reasonable conjecture we now propose that curved space-time, like an inflexible sheet wrapped around a curved surface, must develop persistent wrinkles - the elementary units of matter or energy. We envisage flat space-time in featureless undulation that develops elementary wave packets when curved. We recognize few types of wave packet with internal wave patterns perceived as the characteristic mass, charge, spin and chirality of the four-dimensional elementary units whose behaviour is prescribed by a potential function according to equation (2).

On projection into three-dimensional tangent space the chiral forms known as matter and antimatter are distinct and, as $c \rightarrow \infty$ [28], obey conjugate forms of the wave equation (2a), the three-dimensional approximation of (2). To substantiate this reasoning it is noted that many features of the $\mathrm{H}$ atomic spectrum are reproduced 
to good approximation by the solution of (5) for the single electron on a hydrogen atom.

The crunch comes when trying to analyze non-classical many-electron systems by the same procedure. The mathematics to solve the many-body differential equation does not exist. The popular alternative is to consider each electron as an individual particle and to describe an $n$-electron system by a probability density in $3 n$ dimensional configuration space. The use of complex variables is tacitly avoided. The result is a procedure that pretends to simulate a non-classical problem by a classical model, with an unnecessary complicated structure, designed to resemble quantum formalism. In this case the statistical model that works for an ideal gas, fails to explain the behaviour of a many-electron wave.

An alternative procedure is suggested by the recognition of elementary matter as wave-like distortions of space-time. Unlike free-floating hard particles in a void, the wave packets envisaged here remain part of the medium and their distribution therefore depends on the symmetry of space-time. The extranuclear charge cloud on an atom may thus be viewed as the coalescence of electronic wave packets to constitute a common spherical standing wave around the nucleus. The internal structure of the wave must reflect the charge distribution as optimized under the nuclear attraction, like a three-dimensional analogue of the essentially planar solar system.

Optimization by a golden spiral predicts the correct distribution of matter in the solar sytem [29], with the inference that the spiral structure reflects space-time topology. Fractal models of the universe, which imply cosmic self-similarity, would then indicate the same optimization for extranuclear electron density. The resulting wave structure inevitably carries an imprint of the golden ratio.

Interatomic interaction entails the interference of extranuclear electronic waves. Constructive interference must occur at specific interatomic distances, which should correlate with the notion of bond order, numerically related to the golden ratio. The feasibility of modelling chemical interaction by elementary number theory is foreseen.

\section{Conclusion}

Contrary to popular belief new ideas in science are rarely embraced with acclaim. This was known, in a different context, to Machiavelli who stated 500 years ago [30]:

... that there is nothing more difficult to arrange, more doubtful of success, and more dangerous to carry through than initiating changes .... The innovator makes enemies of all those who prospered under the old order, and only lukewarm support is forthcoming from those who would prosper under the new. Their support is lukewarm partly from fear of their adversaries, who have the existing laws on their side, and partly because men are generally incredulous, never really trusting new things unless they have tested them by experience. In consequence, whenever those who oppose the changes can do so, they attack vigorously, and the defence made by the others is only lukewarm. So both the innovator and his friends are endangered together. 
As an example in science, Schrödinger had to endure such attacks in response to his wave-mechanical interpretation of quantum effects, as evidenced by his statement, from the biography by Moore [31]:

Let me say at the outset, that in this discourse, I am opposing not a few special statements of quantum mechanics held today (1950), I am opposing as it were the whole of it, I am opposing its basic views that have been shaped 25 years ago, when Max Born put forward his probability interpretation, which was accepted by almost everybody. ... I don't like it, and I'm sorry I ever had anything to do with it.

Schrödinger tried to introduce a wave model of matter in opposition to the contemporary dogmatic belief in elementary particles. He failed and the debate was closed for the best part of a century. By now the ranks of those who profit by the preservation of the wave-particle model have swelled by orders of magnitude. Even the few lukewarm defenders have to rely on commercialized software based on probabilities to generate results considered suitable for publication in the mainstream media. There is no reason to believe that the 4D analysis proposed here will be treated any differently. Promoting a new system remains fraught with danger, despite compelling mathematical support.

Current consensus among mathematicians [32] is that normed division algebras, which could be the basis of high-dimensional physical vector spaces, are restricted to $1,2,4$ and 8 dimensions. We interpret this to explain why physical theories in 3 dimensions are plagued by confusing features such as non-commutation and complex phases that intrude themselves in quantum theory. In four dimensions these would be natural features. The same argument explains why five-dimensional Kaluza-Klein models fail as unified field theories. The alternative four-dimensional unification based on projective geometry in curved space-time [33] and a wave model of matter works without awkward compacted dimensions and develops gauge invariance in a natural way.

Some common practices further aggravate the situation. The accepted interpretation of special relativity considers all space outside of the Minkowski time cone as non-physical. This prejudice obscures the non-local nature of quantum theory and distorts the common perception of space-time topology. By an equally arbitrary assumption advanced solutions (in $-t$ ) of the three-dimensional wave equation are rejected. This way all perceptions of space-time chirality, the existence of antimatter and non-local correlation are lost.

In chemical theory misreading of the superposition principle underpins the widespread use of real orbitals and basis sets, without any mathematical meaning. Half a century's research results in quantum chemistry may well be wasted effort. But this represents Machiavelli's profit under the old system. We propose that the utility of number theory in the description of chemical systems could provide an escape route from this dilemma.

Acknowledgements I have discussed the mathematics of four dimensions many times with my colleague Casper Schutte and I gratefully acknowledge his valuable input. 


\section{Appendix}

\section{Hypercomplex Numbers}

The theory of Special Relativity is conveniently summarized by a set of equations, known as a Lorentz transformation, which describes all relative motion, including that of electromagnetic signals, observed to propagate with constant speed $c$, irrespective of the observer's state of motion. This transformation,

$$
x^{\prime}=\frac{x-v t}{\sqrt{1-v^{2} / c^{2}}} \quad, \quad t^{\prime}=\frac{t-v x / c^{2}}{\sqrt{1-v^{2} / c^{2}}},
$$

relates two frames of reference in relative motion and has no meaning in a system that separates space and time variables. The resulting four-dimensional space-time is known as Minkowski space.

From the equation for the moving front of a spherical light wave:

$$
x^{2}+y^{2}+z^{2}=c^{2} t^{2}
$$

a fourth coordinate is often defined as $x_{0}=c t$, redefined by Minkowski as $x_{0}=i c t$, such that

$$
x_{0}^{2}+x_{1}^{2}+x_{2}^{2}+x_{3}^{2}=0
$$

at the wavefront. In terms of the velocity ratio $\beta=v / c$ and $\gamma=1 / \sqrt{1-\beta^{2}}$, the Lorentz transformation for uniform relative motion along $x_{1}$ then takes the simple form, $\mathbf{x}^{\prime}=\mathbf{L x}$ i.e.

$$
\left(\begin{array}{c}
x_{0}^{\prime} \\
x_{1}^{\prime}
\end{array}\right)=\left(\begin{array}{cc}
\gamma & -i \beta \gamma \\
i \beta \gamma & \gamma
\end{array}\right)\left(\begin{array}{l}
x_{0} \\
x_{1}
\end{array}\right)
$$

This transformation matrix has the same form as an orthogonal rotation matrix

$$
\mathbf{R}=\left(\begin{array}{rr}
\cos \phi & -\sin \phi \\
\sin \phi & \cos \phi
\end{array}\right)
$$

The Lorentz transformation thereby defines a rotation in the $(x, t)$ plane through an imaginary angle $\phi$, defined by

$$
\cos \phi=\frac{1}{\sqrt{1-\beta^{2}}} \quad, \quad \sin \phi=\frac{i \beta}{\sqrt{1-\beta^{2}}} \quad, \quad \phi=\tan ^{-1} i \beta .
$$

As this rotation mathematically interchanges time and space coordinates it means that they are symmetry related and no longer seperable in the usual way. It is therefore more appropriate to deal with four-dimensional space-time, rather than the traditional three-dimensional space and absolute time. To visualize Minkowski space it is useful first to review some properties of the complex plane. 


\subsection{Complex Numbers}

There is a similarity between two-dimensional vectors and complex numbers, but also subtle differences. One striking difference is between the product functions of complex numbers and vectors.

The product of two vectors is either a scalar

$$
z_{3}=\mathbf{z}_{1} \cdot \mathbf{z}_{2}=z_{1} z_{2} \cos \theta=x_{1} x_{2}+y_{1} y_{2}
$$

or a vector

$$
\mathbf{z}_{3}=\mathbf{z}_{1} \times \mathbf{z}_{2}=z_{1} z_{2} \sin \theta=y_{1} x_{2}-y_{2} x_{1} .
$$

By way of comparison, the product of two complex numbers is

$$
\begin{aligned}
z_{1} z_{2} & =\left(x_{1}+i y_{1}\right)\left(x_{2}+i y_{2}\right) \\
& =\left(x_{1} x_{2}-y_{1} y_{2}\right)+i\left(x_{1} y_{2}+x_{2} y_{1}\right) \\
z_{3} & =x_{3}+i y_{3} .
\end{aligned}
$$

The complex product contains two terms, not unlike the scalar and vector products, from which it differs only in a sign convention.

It is well known that the complex number $x+i y$ is given in polar form by Euler's equation:

$$
r(\cos \theta+i \sin \theta)=r e^{i \theta}
$$

The product

$$
z_{1} z_{2}=r_{1} e^{i \theta_{1}} \cdot r_{2} e^{i \theta_{2}}=r_{1} r_{2} e^{i\left(\theta_{1}+\theta_{2}\right)}
$$

is recognized immediately as the rotation of $z_{1}$ through the angle $\theta_{2}$ and increase of its length by a factor $r_{2}$. To summarize: an operator $w=u+i v=C e^{i \theta}$, with $C^{2}=u^{2}+v^{2}, \theta=\tan ^{-1}(v / u)$, when it multiplies any vector increases its length by a factor $C$. The magnitude and phase of the complex number $x+i y$ are $r=\sqrt{x^{2}+y^{2}}$ and $\theta=\tan ^{-1}(y / x)$. The complex conjugate $z^{*}=(x+i y)^{*}=x-i y$ has the phase $-\theta$ and magnitude $r$. The magnitude of a complex quantity is obtained from $z z^{*}=$ $\left(r e^{i \theta}\right)\left(r e^{-i \theta}\right)=r^{2}$, which is always real and nonnegative.

Matrices in the form $\left(\begin{array}{cc}\alpha & -\beta \\ \beta & \alpha\end{array}\right)$, combined by matrix addition and multiplication are isomorphic with the field of complex numbers $(\alpha+i \beta)$. This way,

$$
(\alpha+i \beta)+(\gamma+i \delta)=(\alpha+\gamma)+i(\beta+\delta)
$$

and

$$
\left(\begin{array}{cc}
\alpha & -\beta \\
\beta & \alpha
\end{array}\right)+\left(\begin{array}{cc}
\gamma & -\delta \\
\delta & \gamma
\end{array}\right)=\left(\begin{array}{cc}
\alpha+\gamma & -(\beta+\delta) \\
\beta+\delta & \alpha+\gamma
\end{array}\right)
$$

Also

$$
(\alpha+i \beta)(\gamma+i \delta)=(\alpha \gamma-\beta \delta)+i(\beta \gamma+\alpha \delta)
$$

and 


$$
\left(\begin{array}{cc}
\alpha & -\beta \\
\beta & \alpha
\end{array}\right)\left(\begin{array}{cc}
\gamma-\delta \\
\delta & \gamma
\end{array}\right)=\left(\begin{array}{cc}
\alpha \gamma-\beta \delta & -(\beta \gamma+\alpha \delta) \\
\beta \gamma+\alpha \delta & \alpha \gamma-\beta \delta
\end{array}\right)
$$

Euler's equation in the form:

$$
r e^{i \theta}=r\left(\begin{array}{rr}
\cos \theta & -\sin \theta \\
\sin \theta & \cos \theta
\end{array}\right)
$$

confirms the geometrical meaning of complex numbers as rotation and enlargement. As an example the complex number $i$ corresponds to the matrix $\left(\begin{array}{rr}0 & -1 \\ 1 & 0\end{array}\right)$, which represents a counter-clockwise rotation of $\pi / 2$ about the origin.

Another alternative form of the rotation matrix is obtained by setting $\alpha=a^{2}+b^{2}$ and $\beta=2 a b$, with $a=\cos (\theta / 2), b=\sin (\theta / 2)$. Hence

$$
\begin{aligned}
& \alpha=\cos ^{2}(\theta / 2)-\sin ^{2}(\theta / 2)=\cos \theta \\
& \beta=2 \cos (\theta / 2) \sin (\theta / 2)=\sin \theta,
\end{aligned}
$$

i.e.

$$
(a, b)=\left(\begin{array}{cc}
a^{2}-b^{2} & -2 a b \\
2 a b & a^{2}-b^{2}
\end{array}\right)=\left(\begin{array}{rr}
\cos \theta & -\sin \theta \\
\sin \theta & \cos \theta
\end{array}\right)
$$

\subsection{Quaternions}

Extension of the complex formalism to more dimensions suggests the definition of related hypercomplex numbers. On multiplication of two three-dimensional vectors, without defining the mathematical properties of unit vectors $\mathbf{i}, \mathbf{j}, \mathbf{k}$, the formal result is:

$$
\begin{aligned}
q & =\left(\mathbf{i} x_{1}+\mathbf{j} y_{1}+\mathbf{k} z_{1}\right)\left(\mathbf{i} x_{2}+\mathbf{j} y_{2}+\mathbf{k} z_{2}\right) \\
& =\mathbf{i}^{2} x_{1} x_{2}+\mathbf{j}^{2} y_{1} y_{2}+\mathbf{k}^{2} z_{1} z_{2} \\
& +\mathbf{i j} x_{1} y_{2}+\mathbf{j} \mathbf{i} y_{1} x_{2}+\mathbf{i k} x_{1} z_{2}+\mathbf{k i} z_{1} x_{2}+\mathbf{j} \mathbf{k} y_{1} z_{2}+\mathbf{k j} z_{1} y_{2}
\end{aligned}
$$

This expression is rearranged into the same form as a complex product by defining

$$
\begin{aligned}
& \mathbf{i}^{2}=\mathbf{j}^{2}=\mathbf{k}^{2}=-1 \\
& \mathbf{i j}=\mathbf{k}, \mathbf{j k}=\mathbf{i}, \mathbf{k i}=\mathbf{j} \\
& \mathbf{j i}=-\mathbf{k}, \mathbf{k} \mathbf{j}=-\mathbf{i}, \mathbf{i k}=-\mathbf{j}
\end{aligned}
$$

the result first obtained by William Hamilton who defined

$$
q=-\left(x_{1} x_{2}+y_{1} y_{2}+z_{1} z_{2}\right)+i\left(y_{1} z_{2}-y_{2} z_{1}\right)+j\left(z_{1} x_{2}-z_{2} x_{1}\right)+k\left(x_{1} y_{2}-x_{2} y_{1}\right)
$$


with the rule of composition: $i^{2}=j^{2}=k^{2}=i j k=-1$.

A hypercomplex number of unit norm can now be defined in the form $z=a_{0}+a_{i} e_{i}$, where the $e_{i}$ are generalizations of $\sqrt{-1}$, in matrix notation:

$$
1=\left(\begin{array}{ll}
1 & 0 \\
0 & 1
\end{array}\right), \quad e_{1}=\left(\begin{array}{cc}
i & 0 \\
0 & -i
\end{array}\right), \quad e_{2}=\left(\begin{array}{cc}
0 & 1 \\
-1 & 0
\end{array}\right), \quad e_{3}=\left(\begin{array}{cc}
0 & i \\
i & 0
\end{array}\right)
$$

For $i=1,3$ it is called a quaternion and the matrices are quaternion units. The unit number 1 represents the unit vector in the fourth dimension.

To represent a quaternion in matrix form, as was done for complex numbers, it is written as a pair of complex numbers, $q=(u, v)$, in the same way that the complex number $a+i b$ is written as $(a, b)=\left(\begin{array}{rr}a & -b \\ b & a\end{array}\right)$. As for complex numbers the product of $(u, v)$ and its conjugate should be $\left(|u|^{2}+|v|^{2}, 0\right)$. This result is obtained by defining the conjugate $q^{*}=\left(u^{*},-v\right)$ and the matrix equivalent of $(u, v)$ as $\left(\begin{array}{cc}u v^{*} \\ -v & u^{*}\end{array}\right)$. This way

$$
\begin{aligned}
q q^{*}=\left(\begin{array}{cc}
u & v^{*} \\
-v & u^{*}
\end{array}\right)\left(\begin{array}{cc}
u^{*} & -v \\
v & u
\end{array}\right) & =\left(\begin{array}{cc}
u u^{*}+v^{*} v & -u v^{*}+v^{*} u \\
-v u^{*}+u^{*} v & v v^{*}+u^{*} u
\end{array}\right) \\
& \equiv\left(|u|^{2}+|v|^{2}, 0\right)
\end{aligned}
$$

The modulus of $q, \sqrt{q^{*} q}=\sqrt{u^{2}+v^{2}}$. Notice that the determinant of the matrix of $q=(u, v)$, gives

$$
\left|\begin{array}{rr}
u & v^{*} \\
-v & u^{*}
\end{array}\right|=|u|^{2}+|v|^{2}
$$

By expanding the quaternion in terms of the complex pair, $u=a+i b$ and $v=c+i d$, it follows directly that $q^{2}=a^{2}+b^{2}+c^{2}+d^{2}$. In terms of four quaternion components:

$$
q \cdot q=q_{0}^{2}+q_{1}^{2}+q_{2}^{2}+q_{3}^{2} .
$$

For $|u|^{2}+|v|^{2}=1$, the product of the quaternion and its conjugate (or adjoint) is the unitary matrix $\left(\begin{array}{ll}1 & 0 \\ 0 & 1\end{array}\right)$. Unit quaternions (of modulus 1 ) are therefore seen to define rotations of $\mathbb{C}^{2}$, just as the unit complex numbers correspond to rotations of $\mathbb{R}^{2}$. The group of unitary transformations of $\mathbb{C}^{2}$ of determinant 1 is the special unitary Lie group $S U(2)$, which describes the spin function.

In Hamilton's notation the conjugate of the general quaternion $q=a+i b+j c+$ $k d$ is $q^{*}=a-i b-j c-k d$, so that the square of the magnitude, $|q|^{2}=q^{*} q=a^{2}+$ $b^{2}+c^{2}+d^{2}$ is a simple extension of the rule for complex numbers.

As with complex numbers the product of two quaternions

$$
\begin{aligned}
(p=\alpha+i \beta+j \gamma+k \delta) q & =(\alpha a-\beta b-\gamma c-\delta d)+i(\alpha b+\beta a+\gamma d-\delta c) \\
& +j(\alpha c-\beta d+\gamma a+\delta b)+k(\alpha d+\beta c-\gamma b+\delta a)
\end{aligned}
$$


is another quaternion. Quaternion multiplication is clearly not commutative, as $p q \neq$ qp.

By expanding the exponential and using the rules for multiplication of $i, j, k$, it can be shown that, for $\alpha^{2}+\beta^{2}+\gamma^{2}=1$, a quaternion equivalent of Euler's equation for the imaginary exponent is obtained as:

$$
e^{\theta(i \alpha+j \beta+k \gamma)}=\cos \theta+\sin \theta(i \alpha+j \beta+k \gamma) .
$$

This exponential is of unit length; the exponent represents a space vector of length $\theta$, with direction as given by the direction cosines $\alpha, \beta, \gamma$.

Any quaternion can now be represented in the form $Q e^{\theta(i \alpha+j \beta+k \gamma)}$, where $Q$ is the length of a four vector with direction as fixed by $\theta, \alpha, \beta, \gamma$. By analogy with the complex exponential it is anticipated that this operator (with $Q=1$ ) describes a rotation. The argument on which it operates is also a quaternion. In three dimensions the vector $f=i x+j k+k z$, in quaternion notation, is rotated by angle $\theta$ about an axis of direction cosines $\alpha, \beta, \gamma$ into $f^{\prime}$ according to:

$$
f^{\prime}=i x^{\prime}+j y^{\prime}+k z^{\prime}=e^{(\theta / 2)(i \alpha+j \beta+k \gamma)} f e^{-(\theta / 2)(i \alpha+j \beta+k \gamma)}=q \cdot f \cdot q^{-1},
$$

where $\alpha^{2}+\beta^{2}+\gamma^{2}=1$.

As in (6) we define the rotation matrix by a pair of complex numbers $(u, v)$, setting

$$
\begin{aligned}
& q_{0}=a \cos (\theta / 2) \\
& q_{1}=i \sin (\theta / 2) \\
& q_{2}=j \sin (\theta / 2) \\
& q_{3}=k \sin (\theta / 2),
\end{aligned}
$$

which define the three-dimensional rotation matrix:

$$
\mathbf{R}(q)=\left(\begin{array}{ccc}
q_{0}^{2}+q_{1}^{2}-q_{2}^{2}-q_{3}^{2} & 2 q_{1} q_{2}-2 q_{0} q_{3} & 2 q_{1} q_{3}+2 q_{0} q_{2} \\
2 q_{1} q_{2}+2 q_{0} q_{3} & q_{0}^{2}-q_{1}^{2}+q_{2}^{2}-q_{3}^{2} & 2 q_{2} q_{3}-2 q_{0} q_{1} \\
2 q_{1} q_{3}-2 q_{0} q_{2} & 2 q_{2} q_{3}+2 q_{0} q_{1} & q_{0}^{2}-q_{1}^{2}-q_{2}^{2}+q_{3}^{2}
\end{array}\right)
$$

Each row (or column) has unit magnitude, e.g.:

$$
\left(q_{0}^{2}+q_{1}^{2}-q_{2}^{2}-q_{3}^{2}\right)^{2}+\left(2 q_{1} q_{2}-2 q_{0} q_{3}\right)^{2}+\left(2 q_{1} q_{3}+2 q_{0} q_{2}\right)^{2}=(q \cdot q)^{2}=1,
$$

and the total matrix $\left(\mathbf{R} f=q f q^{-1}\right)$ is orthogonal, as required for 3D rotation.

Quaternions describe rotation in any number of dimensions from 1 to 4 . It is straightforward to demonstrate ${ }^{3}$ that for the special case of rotation about the $x$ axis:

$$
f^{\prime}=e^{(\theta / 2) i}(i x+j y+k z) e^{-(\theta / 2) i}
$$

${ }^{3}$ Using: $\sin (\theta / 2)= \pm \sqrt{\frac{1}{2}(1-\cos \theta)} \quad, \quad \cos (\theta / 2)= \pm \sqrt{\frac{1}{2}(1+\cos \theta)}$ 


$$
\begin{aligned}
& =i x+j(y \cos \theta-z \sin \theta)+k(y \sin \theta+z \cos \theta) \\
& =\left(\begin{array}{lll}
i & j & k
\end{array}\right)\left(\begin{array}{ccc}
1 & 0 & 0 \\
0 & \cos \theta & -\sin \theta \\
0 & \sin \theta & \cos \theta
\end{array}\right)\left(\begin{array}{l}
x \\
y \\
z
\end{array}\right)
\end{aligned}
$$

i.e. a rotation through the imaginary angle $\theta$ about the $x$-axis in the $(y, z)$-plane. The right-to-left order of matrix multiplication is important. Proof of the general case only involves more algebra. Symbolically $f^{\prime}=\zeta f \zeta^{*}$. By a second rotation $f^{\prime \prime}=\eta f^{\prime} \eta^{*}=\eta \zeta f \zeta^{*} \eta^{*}$.

The final result depends on the order in which the operations are applied, because of the fact that the quaternions $\eta$ and $\zeta$ do not commute. The quantity $Q$ is called the tensor (stretcher) and the exponential is called the versor (turner) of the operator.

In four-dimensional rotation the argument of the operation is the full quaternion four-vector, $v_{\mu}=\left(v_{0}, v_{i}\right)$, rather than the three-vector $f$ with $v_{0}=0$, considered before. On working out the full rotation matrix it turns out to decompose into a pair of $3 \mathrm{D}$ rotations, such as (8), indicating that the four-dimensional rotation amounts to double covering of the underlying space of 3D rotations [35]. 


\section{References}

1. Dirac PAM (1928) Proc Roy Soc A 117:610.

2. Taub AH, Veblen O, von Neumann J (1934) Proc Nat Acad Sci US 20:383

3. Dirac PAM (1938) Proc Roy Soc A167:148.

4. Morse PM, Feshbach H (1956) Methods of Theoretical Physics. McGraw-Hill, NY.

5. Misner CW, Thorne K, Wheeler JA (1973) Gravitation. Freeman, San Francisco, p. 1143.

6. Boeyens JCA (2008) Chemistry from First Principles. Springer.com.

7. Batty-Pratt EP, Racey TJ (1980) Int J Theor Phys 19: 437.

8. Carmeli M (1985) Nuovo Cimento Lett 42:67.

9. Lévy-Leblond J-M (1967) Comm math Phys 6:286.

10. Pauli W (1927) Z für Physik 43:601.

11. Kauffman LH (1987) On Knots, Princeton Univ Press, NJ.

12. Unsöld A (1927) Ann. d. Physik 82:355.

13. Bohm D (1996) The Special Theory of Relativity. Routledge, London.

14. Einstein A. (1911) On the influence of gravitation on the propagation of light. Translation of German original, in [15].

15. Perrett P, Jeffery GB (1952) (translators). The Principle of Relativity. Dover, N.Y.

16. Jennison RC, Drinkwater AJ (1977) J Phys A 10:167.

17. Elbaz C (1986) Phys Lett 114A:445.

18. Elbaz C (1983) CR Acad Sc Paris 297:455.

19. Feinberg G (1967) Phys Rev 159:1089.

20. Corben HC (1977) Lett Nuovo Cimento 20:645.

21. Wolff M (1993) Physics Essays 6:181; - (1995) Galilean Electrodynamics Sept/Oct:83; - (1997) Frontier Perspectives 4:67.

22. Jennison RC (1978) J Phys A: Math Gen 11:1525.

23. Mc Crea WH (1937) Supplement to Nature of June 12, 139:1002. (Quoting Veblen and Whitehead)

24. Bohm D (1951) Quantum Theory. Dover reprint, Prentice-Hall, NJ.

25. de Broglie L (1926) Nature 118:441.

26. Schrödinger E (1978) Collected papers on Wave Mechanics, Translated from the 1927 German edition, Chelsea, NY, p. 124.

27. Bohm D, Bub J (1966) Revs Mod Phys 38:453.

28. Oppenheimer JR (1930) Phys Rev 35:461.

29. Boeyens JCA (2009) Physics Essays 22:493.

30. Machiavelli N (1975) The Prince. Penguin Books, Hammondsworth. Translated from the 1929 Italian edition by George Bull.

31. Moore W (1989) Schrödinger - Life and Thought. University Press, Cambridge.

32. Gowers T (ed.) (2008) The Princeton Companion to Mathematics. University Press, Princeton.

33. Veblen O (1933) Projektive Relativitätstheorie. Springer, Berlin. English translation in [34].

34. Boeyens JCA (2010) Chemical Cosmology. Springer.com.

35. A.J. Hanson, Visualizing Quaternions, Elsevier, Amsterdam, (Morgan Kaufman), (2006). 